\title{
Fast Algorithm for Three-Dimensional Single Near-Field Source Localization with Uniform Circular Array
}

\author{
Yuntao Wu*, Hai Wang*, Longting Huang*, H.C.So ${ }^{\dagger}$ \\ ${ }^{*}$ Key Laboratory of Intelligent Robot in Hubei Province \\ Wuhan Institute of Technology, Wuhan, 430073, China \\ ${ }^{\dagger}$ Department of Electronic Engineering \\ City University of Hong Kong, Kowloon, Hong Kong
}

\begin{abstract}
A least squares estimation algorithm for the azimuth and elevation angles as well as range of a single near-field source impinging on a uniform circular array is proposed. By exploiting the centro-symmetric array configuration, two correlation functions are devised to obtain their closed-form estimates. It is shown that the proposed method gives comparable estimation performance with that of the conventional MUSIC scheme but the former is much computationally simple.
\end{abstract}

Keywords: Near-field source localization, two-dimensional angle estimation, range estimation, uniform circular array.

\section{Introduction}

Passive source localization with an array of spatially separated sensors with a priori known positions has many important application areas such as radar, sonar and wireless communications. For three-dimensional (3-D) positioning, uniform circular array (UCA) is preferable over the rectangular and nonuniform arrays because of its advantages of providing $360^{\circ}$ azimuthal coverage, almost unchanged directional pattern and additional elevation angle information [1][2]. In this Letter, we address the problem of finding the spherical coordinates of a narrow-band near-field source with an UCA. A standard solution is to use the 3-D MUSIC method [1] for joint estimation of the azimuth and elevation angles as well as range, which implies a very high implementation cost. Via employing an additional sensor at the UCA center, Bae et al. [3] have recently developed a computationally attractive least squares (LS) positioning algorithm but it only works for a noncircular source. Similar to [3], we propose a fast LS source localization algorithm which first computes the azimuth and elevation angle estimates, followed by range estimation. Unlike [3], the center reference sensor is not required and the source is not restricted to be noncircular. The key idea in the algorithm development is to utilize the UCA centro-symmetry to design two correlation sequences whose phases contain the position information. It is noteworthy that the devised method can be considered as a generalization of our far-field localization scheme developed in [2] where there is no range parameter. Computer simulations show that the proposed LS algorithm is comparable to the 3-D MUSIC method in terms of estimation performance but is more computationally simple as 3-D search and eignenvalue decomposition are not required.

\section{Format instructions}

A centro-symmetric circular array with radius $R$ and $M$ omnidirectional sensors, where $M$ is assumed an integral multiple of 4, is employed. Without loss of generality, we assign all sensors on the $x y$ - plane and the UCA center as the origin of the coordinate system. Consider the narrow-band near-field source is located at $(\theta, \phi, r)$ in the spherical coordinate system, where $\theta \in[0,2 \pi)$ is the azimuth angle measured counterclockwise from the $x$ axis, $\phi \in[0, \pi / 2)$ is the elevation angle measured downward from the $z$ axis, and $r$ is the range measured from the UCA center. The output of the $k$ th sensor of the UCA is [1]:

$$
\begin{gathered}
x_{k}(n)=s(n) e^{j \frac{2 \pi}{\lambda}\left(r-r_{k}(\theta, \phi, r)\right)}+w_{k}(n) \\
k=1,2, \cdots, M, n=1,2, \cdots, N
\end{gathered}
$$

where represents the zero-mean complex narrow-band source signal whose power is $E\left\{|s(n)|^{2}\right\}=\sigma_{s}^{2}$ with source signal whose power is $E\left\{|s(n)|^{2}\right\}=\sigma_{s}^{2}$ with $E$ being the expectation operator, while each of the measurement noises $\left\{w_{k}(n)\right\}$ is assumed to be a second-order ergodic, zero-mean, spatially and temporally white complex Gaussian process, which is independent of $s(n)$. Each sensor receives $N$ samples, $\lambda$ is the wavelength of the source and $r_{k}(\theta, \phi, r)$ is the distance between the source and the $k$ th sensor, which has the form of

$$
r_{k}(\theta, \phi, r)=\sqrt{r^{2}+R^{2}-2 r R \xi_{k}(\theta, \phi)}, k=1,2, \cdots, M
$$

where

$$
\xi_{k}(\theta, \phi)=\cos \left(\gamma_{k}-\theta\right) \sin (\phi)
$$

with $\gamma_{k}=2 \pi(k-1) / M$ being the angle of the $k$ th sensor measured counterclockwise from the $x$-axis. Given $\left\{x_{k}(n)\right\}$, our task is to estimate $\theta, \phi, r$.

For a sufficiently large value of $r$ and according to Taylor series expansion, (2) is well approximated as [1]:

$$
r_{k}(\theta, \phi, r) \approx r-R \xi_{k}(\theta, \phi)+\frac{R^{2}}{2 r}\left(1-\xi_{k}^{2}(\theta, \phi)\right)
$$

Substituting (4) into (1), we get:

$$
x_{k}(n)=s(n) e^{j \frac{2 \pi R}{\lambda}\left[\xi_{k}(\theta, \phi)-\frac{R}{2 r}\left(1-\xi_{k}^{2}(\theta, \phi)\right)\right]}+w_{k}(n)
$$


From the centro-symmetric array configuration with even number of sensors, we have $\gamma_{M / 2+k}=\gamma_{k}+\pi$ which gives:

$$
a_{k}(\theta, \phi)=a_{k+M / 2}^{*}(\theta, \phi), k=1,2, \cdots, M / 2
$$

where $a_{k}(\theta, \phi)$ denotes the complex array response of the signal at the $k$ th sensor and $*$ is the complex conjugation operator. On the other hand, we also have $\gamma_{M / 4+k}=\gamma_{k}+\pi / 2$ to yield:

$$
\begin{array}{r}
\xi_{k+M / 4}(\theta, \phi, r)=-\sin \left(\gamma_{k}-\theta\right) \sin (\phi), \\
k=1,2, \cdots, 3 M / 4
\end{array}
$$

Based (6) and (7), we construct two autocorrelation sequences with the use of $\left\{x_{k}(n)\right\}$ :

$$
p(k)=\frac{1}{N} \sum_{n=1}^{N} x_{k}(n) x_{k+M / 2}^{*}(n), k=1,2, \cdots, M / 2
$$

and

$$
q(k)=\frac{1}{N} \sum_{n=1}^{N} x_{k}(n) x_{k+M / 4}^{*}(n), k=1,2, \cdots, 3 M / 4
$$

when both $N$ and signal-to-noise ratio (SNR) are sufficiently large and assuming that ergodicity holds, $p(k)$ can be approximated as [2]:

$$
\begin{aligned}
p(k) \approx & E\left\{x_{k}(n) x_{k+M / 2}^{*}(n)\right\} \\
= & \sigma_{s}^{2} a_{k}(\theta, \phi) a_{k+M / 2}^{*}(\theta, \phi) \\
= & \sigma_{s}^{2} e^{j \frac{4 \pi R}{\lambda} \cos \left(\theta-\gamma_{k}\right) \sin (\phi)} \\
& k=1,2, \cdots, M / 2
\end{aligned}
$$

Similarly, (9) has the following approximation:

$$
\begin{gathered}
q(k) \approx E\left\{x_{k}(n) x_{k+M / 4}^{*}(n)\right\} \\
=\sigma_{s}^{2} a_{k}(\theta, \phi) a_{k+M / 4}^{*}(\theta, \phi) \\
=\sigma_{s}^{2} e^{j \frac{4 \pi R}{\lambda}\left[\sqrt{2} \cos \left(\theta-\gamma_{k}-\pi / 4\right) \sin (\phi)\right.} \\
\quad+\frac{R}{2 r} \cos \left(2 \theta-2 \gamma_{k}\right) \sin ^{2}(\phi) \\
k=1,2, \cdots, 3 M / 4
\end{gathered}
$$

We will first estimate $\theta$ and $\phi$ from (8) and then employ (9) for range estimation with the use of the angle estimates as follows. Let $\omega_{k}$ be the phase angle of $p(k)$. From (10), we obtain:

$$
\begin{gathered}
\omega_{k} \approx \frac{4 \pi R}{\lambda} \cos \left(\theta-\gamma_{k}\right) \sin (\phi)+2 \pi m_{k}, \\
k=1,2, \cdots, M / 2
\end{gathered}
$$

where $m_{k}$ is an integer. Since the phase ambiguity problem has been well addressed in the literature, the interested reader is referred to [4][5] for determining $m_{k}$. In the following, we assume that the phase ambiguity has been successfully solved which leads to a unique angle estimation. Without loss of generality, we assign $m_{k}=0$ and rewrite (12) as:

$$
\begin{aligned}
& \omega_{k} \approx \frac{4 \pi R}{\lambda}\left[\begin{array}{ll}
\cos \left(\gamma_{k}\right) & \sin \left(\gamma_{k}\right)
\end{array}\right]\left[\begin{array}{c}
\sin (\phi) \cos (\theta) \\
\sin (\phi) \sin (\theta)
\end{array}\right], \\
& k=1,2, \cdots, M / 2
\end{aligned}
$$

Expressing (13) in matrix form yields

$$
\mathbf{w} \approx \mathbf{A b}
$$

where

$$
\mathbf{w}=\left[\begin{array}{llll}
\omega_{1} & \omega_{2} & \cdots & \omega_{M / 2}
\end{array}\right]^{T}
$$

$$
\mathbf{A}=\left[\begin{array}{cc}
\cos \left(\gamma_{1}\right) & \sin \left(\gamma_{1}\right) \\
\cos \left(\gamma_{2}\right) & \sin \left(\gamma_{2}\right) \\
\vdots & \vdots \\
\cos \left(\gamma_{M / 2}\right) & \sin \left(\gamma_{M / 2}\right)
\end{array}\right]
$$

and

$$
\mathbf{b}=\frac{4 \pi R}{\lambda}[\sin (\phi) \cos (\theta) \quad \sin (\phi) \sin (\theta)]^{T}
$$

with $T$ denotes the transpose operator. The LS estimate of $\mathbf{b}$, denoted by $\hat{\mathbf{b}}$, is

$$
\hat{\mathbf{b}}=\left[\begin{array}{ll}
\hat{b}_{1} & \hat{b}_{2}
\end{array}\right]^{T}=\left(\mathbf{A}^{T} \mathbf{A}\right)^{-1} \mathbf{A}^{T} \mathbf{w}
$$

The estimate of the angle pair $(\theta, \varphi)$, denoted by $(\hat{\theta}, \hat{\varphi})$, is then determined from $\hat{\mathbf{b}}$ :

$$
\hat{\theta}=\tan ^{-1}\left(\frac{\hat{b}_{2}}{\hat{b}_{1}}\right)
$$

and

$$
\hat{\phi}=\sin ^{-1}\left(\frac{\lambda}{4 \pi R} \sqrt{\hat{b}_{1}^{2}+\hat{b}_{2}^{2}}\right)
$$

Note that the angle estimation procedure in (12)--(17) is identical to that in [2][3]. Using the angle estimates, and denoting the phase of $q(k)$ by $u_{k}$, we obtain the following relationship from (11):

$$
\begin{gathered}
u_{k} \approx \frac{2 \pi R}{\lambda}\left[\sqrt{2} \cos \left(\hat{\theta}-\gamma_{k}-\pi / 4\right) \sin (\hat{\phi})\right. \\
\left.+\frac{R}{2 r} \cos \left(2 \hat{\theta}-2 \gamma_{k}\right) \sin ^{2}(\hat{\phi})\right] \\
k=1,2, \cdots, 3 M / 4
\end{gathered}
$$

assuming that the phase ambiguity problem is fixed. Expressing (18) in matrix form yields:

$$
\mathbf{u} \approx \mathbf{d}-\frac{R}{2 r} \mathbf{v}
$$

where

$$
\begin{aligned}
& \mathbf{u}=\left[\begin{array}{llll}
u_{1} & u_{2} & \cdots & u_{3 M / 4}
\end{array}\right]^{T} \\
& \mathbf{d}=\sin (\hat{\phi})\left[\sqrt{2} \cos \left(\hat{\theta}-\gamma_{1}-\pi / 4\right) \quad \sqrt{2} \cos \left(\hat{\theta}-\gamma_{2}-\pi / 4\right)\right. \\
& \left.\cdots \sqrt{2} \cos \left(\hat{\theta}-\gamma_{3 M / 4}-\pi / 4\right)\right]^{T}
\end{aligned}
$$

and

$$
\begin{aligned}
\mathbf{v}= & \sin ^{2}(\hat{\phi})\left[\cos \left(2 \hat{\theta}-2 \gamma_{1}\right) \quad \cos \left(2 \hat{\theta}-2 \gamma_{2}\right)\right. \\
& \left.\cdots \quad \cos \left(2 \hat{\theta}-2 \gamma_{3 M / 4}\right)\right]^{T}
\end{aligned}
$$

Solving the LS estimate of $1 / r$ according to (19), the estimate of $r$, denoted by $\hat{r}$, is computed using its inverse:

$$
\hat{r}=\frac{R}{2} \mathbf{v}(\mathbf{d}-\mathbf{u})^{T}\left((\mathbf{d}-\mathbf{u})(\mathbf{d}-\mathbf{u})^{T}\right)^{-1}
$$

\section{Simulation Results}

MATLAB simulations have been carried out to evaluate theperformance of the proposed method for 3-D positioning of a single narrow-band near-field source in the presence of additive white Gaussian noise by comparing with the 3-D MUSIC scheme [1]. We consider a UCA consisting of $M=8$ antennas with. $R=\lambda=1$ and the signal is located at $(\theta, \phi, r)=(7 \pi / 18, \pi / 18,2.5 R)$. At each sensor, the number of received samples is $N=100$. We employ the mean square 
error (MSE) as the performance measure and all results provided are averages of 200 independent runs.

Figures 1 to 3 show the MSEs for $\theta, \phi$ and $r$, respectively, versus $\operatorname{SNR} \in[0,25] \mathrm{dB}$. It is seen that both methods give almost the same estimation performance when $\mathrm{SNR} \geq 5 \mathrm{~dB}$.

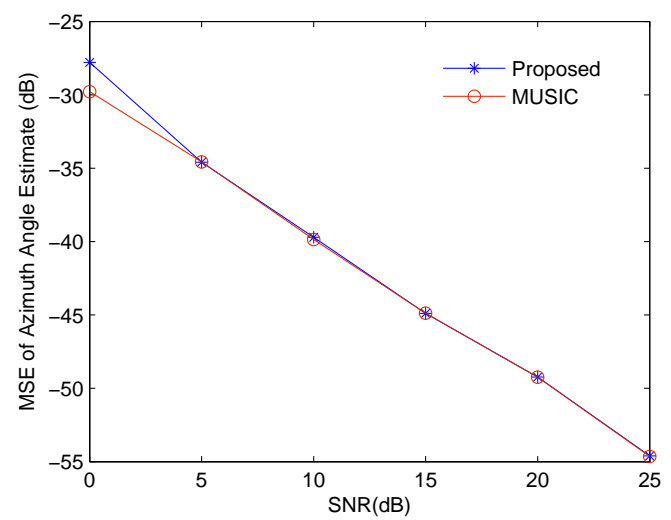

Fig.1. Mean square error for $\theta$ versus SNR

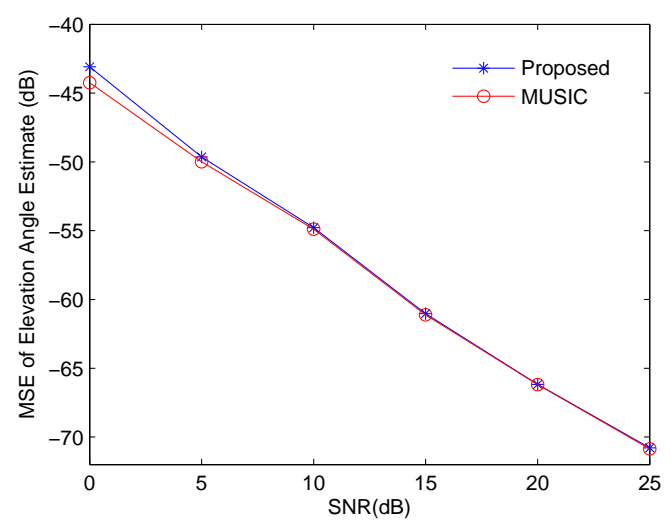

Fig.2. Mean square error for $\phi$ versus SNR

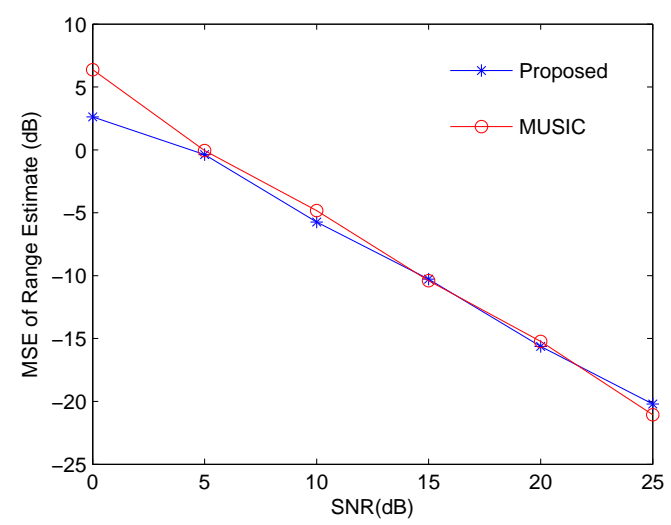

Fig.3. Mean square error for $r$ versus SNR

Regarding the major computational complexity in the proposed method, the number of multiplications for calculating $\hat{\mathbf{b}}$ includes $M N / 2$ in the autocorrelation computation in (8) and $3 M N / 4$ in (9), respectively, and $O\left(M^{2}\right)$ in the LS procedure. On the other hand, the 3-D MUSIC method involves $M^{2} N$ for cross-covariance computation and $O\left(M^{3}\right)$ for the eigenvalue decomposition of the resultant covariance matrix, and additional operations for the 3-D search. Apparently, the computational requirement of the proposed algorithm is much lower than that of the 3-D MUSIC method. The former and latter computation times for a single run are measured as $4.18 \times 10^{-2} \mathrm{~s}$ and $1.5 \mathrm{~s}$, respectively, which agree with our complexity analysis.

\section{Conclusion}

To conclude, a simple closed-form solution for 3-D positioning of a single near-field source using an UCA has been presented. The key idea is to make use of the array centro-symmetric properties to construct correlation functions whose phases contain the information of the azimuth and elevation angles as well as range. In terms of estimation performance, the proposed LS algorithm is comparable to the 3-D MUSIC method but with much smaller computational requirement.

\section{Acknowledgements}

The work described in this paper was jointly supported by a grant from the National Natural Science Foundation of China (No. 60802046) and a grant from the Research Plan Project of Hubei Provincial Department of Education (No. Q20091501).

References

[1] J.H. Lee, D.H. Park, G.T. Park and K.K. Lee, “Algebraic path-following algorithm for localising 3-D near-field sources in uniform circular array," IET Electronics Letters, vol.39, no.17, pp.1283-1285, 2003

[2] Yuntao Wu and H.C. So, "Simple and accurate two dimensional angle estimation for a single source with uniform circular array," IEEE Antennas and Wireless Propagation Letters, vol.7, pp.78-80, 2008

[3] E.H. Bae and K.K. Lee, “Closed-form 3-D localization for single source in uniform circular array with a center sensor," IEICE Trans. on Communications, vol.E92-B, no.3, 2009

[4] M.D. Zoltowski and C.P. Mathews, "'Real-time requency and 2-D angle estimation with sub-Nyquist spatioemporal sampling,” IEEE Trans. Signal Processing, vol.42, no.10, pp.2781-2794, Oct. 1994

[5] K.R. Sundaram, R.K. Mallik and U.M.S. Murthy, "Modulo conversion method for estimating the irection of arrival," IEEE Trans. Aerospace and Electronic Systems, vol.36, no.4, 2000. 